\title{
Method and Effect Evaluation of Implementing Health Management in Physical Examination Centers of 3A Hospitals
}

\author{
Xu Zhen ${ }^{1}$, Yan Bin ${ }^{2}$ \\ ${ }^{1}$ No.303 Hospital of Chinese People's Liberation Army, Nanning, China \\ ${ }^{2}$ No.3 People's Hospital of Nanning, Nanning, China \\ Email address: \\ xzxz90272@163.com (Xu Zhen), 510722381@qq.com (Yan Bin)
}

\section{To cite this article:}

Xu Zhen, Yan Bin. Method and Effect Evaluation of Implementing Health Management in Physical Examination Centers of 3A Hospitals. Science Discovery. Vol. 5, No. 6, 2017, pp. 492-495. doi: 10.11648/j.sd.20170506.27

Received: November 8, 2017; Accepted: November 20, 2017; Published: December 28, 2017

\begin{abstract}
Objective To understand the changes of various routine indices for the people who received health physical examination after the implementation of health management, discuss the method of implementing health management in $3 \mathrm{~A}$ hospitals, provide a reference for better management in 3A hospitals in the future. Methods A total of 1326 people (875 males and 451 females) were selected, whose physical examinations were performed in our hospital's examination center each year in succession from January 2015 to September 2017 and examined items included weight, blood pressure, fasting blood-glucose, blood uric acid and blood lipid. The changes of indices of two examinations before and after implementing health management were compared to evaluate the effects of health management. Results All indices were normal: 192 people in 2015,230 people in 2016, 243 people in 2017; proportion of normal people: $14.48 \%$ in $2015,17.35 \%$ in $2016,18.33 \%$ in 2017 . Difference was statistically significant $(\mathrm{P}<0.01)$. Detection rates for obesity, hypertension, abnormal glucose metabolism, hyperuricemia and hyperlipidemia decreased and bad living behaviors improved obviously. Difference was statistically significant $(\mathrm{P}<0.05)$. Conclusion The health management is based on the health physical examination and implemented in physical examination centers of 3A hospitals, integrates with health education, disease consulting and lifestyle intervention, has high starting point of the platform and flexible, diverse, easy and feasible approaches, is able to raise the examined people's understanding and management of life-style diseases and decrease morbidity.
\end{abstract}

Keywords: General Hospital, Health Management, Effect Evaluation

\section{三甲医院体检中心实施健康管理的方法与效果评价}

许祯 ${ }^{1}$, 颜冰 ${ }^{2}$

${ }^{1}$ 中国人民解放军第三 $\mathrm{O}$ 三医院, 南宁, 中国

2南宁市第三人民医院, 南宁, 中国

邮箱

xzxz90272@163.com（许祯）, 510722381@qq.com（颜冰)

摘要：目的了解健康体检人群在健康管理后各项常规体检指标的变化情况，探讨三甲医院健康管理的方法，为三甲医 院更好地开展健康管理提供参考。方法收集2015年1月～2017年9月三年中每年均在本院健康体检中心进行健康体检， 且体检项目中均包含有体重、血压、空腹血糖、血尿酸和血脂的人员 1326 人（男性 875 人，女性 451 人）每年的健康信 息, 比较健康管理前后两次体检各项指标的变化情况, 评价健康管理效果。结果上述各项体检指标均正常的人数分别 为 2015 年 192 人、 2016 年 230 人、 2017 年 243 人; 正常比例分别为 2015 年 $14.48 \% 、 2016$ 年 $17.35 \% 、 2017$ 年 $18.33 \%$, 比较 
差异具有统计学意义 $(P<0.01)$ 。超重、高血压、糖代谢异常、高尿酸血症、高脂血症的检出率下降, 不良生活行为 明显改善, 差异具有统计学意义 $(P<0.05)$ 。结论三甲医院健康体检中心在健康体检的基础上开展以健康教育、疾病 咨询和生活方式干预为一体的健康管理, 平台起点高, 方法灵活多样且简单易行, 可提高健康体检人群对生活方式性 疾病的认知和管控能力, 降低疾病发生率。

关键词：综合医院，健康管理，效果评价

\section{1. 引言}

健康是指没有疾病，而且身体、心理、社会适宜的 完好状态。健康管理是根据现代生物一心理一社会医学 模式, 应用管理学理论和方法, 对个体或群体的健康进 行全面监测、分析、评估、提供健康咨询和指导, 对健 康危险因素进行干预的全过程。《“健康中国2030”规划 纲要》为推进健康中国建设, 提高人民健康水平指明了 方向。世界卫生组织指出: 在慢性病的形成过程中, $7 \%$ 取决于环境因素， $8 \%$ 取决于医疗条件，10\%取决于社会 因素，15\%取决于遗传因素， $60 \%$ 取决于生活方式。在生 活方式因素中, 膳食不合理、活动不足和吸烟是三大危 险行为因素。通过健康管理, 对人们的不良生活方式进 行干预, 不仅可以提高危险人群的生活质量而且可以降 低卫生服务成本 [1]。健康体检是实现健康管理的关键环 节和重要契机, 在健康体检人群中开展健康管理, 可提 高人群对慢性病及危险因素的知晓力和自我管理能力, 减少危险因素, 有效控制疾病的发生[2]。三甲医院通过 对医疗资源、人力资源、设备与设施、管理与技术等资 源的充分整合与利用, 在场地、设备、人力资源等硬件 基础上, 强化服务理念、技术规范、管理制度、学术创 新和信息技术等软件建设, 可为发展健康管理打造一个 高起点的平台。

本院健康体检中心在健康体检前收集体检者的基本 信息, 包括姓名、性别、年龄、文化程度、婚姻状况、目 前健康状况和既往史、疾病家族史、生活行为、锻炼状况、 饮食习惯等, 体检后综合体检者的体格检查、实验室检查、 影像学检查结果以及个人基本信息为体检者出具详细的 健康体检报告, 在进行健康咨询、健康教育和电话回访指 导、帮助体检者识别健康风险、纠正不良生活习惯、增强 锻炼意识、培养健康理念和健康生活方式的同时, 构建了 和谐的医患关系, 提升了医院的品牌形象。现将做法和成 效报告如下。

\section{2. 资料与方法}

\section{1. 一般资料}

选取2015年1月～2017年9月三年中每年均在本院健 康体检中心进行健康体检的人员 1326 人, 其中男性 875 人, 年龄 $26 \sim 67$ 岁, 平均 $(37.12 \pm 5.84)$ 岁; 女性 451 人, 年龄 $29 \sim 63$ 岁, 平均 $(33.58 \pm 7.91)$ 岁。每年的体检项目均包 含有体重、血压、血糖、血尿酸和血脂。

\section{2. 研究方法}

第一步, 团体体检, 在体检前与参检单位负责人商定 体检套餐, 约定体检时间, 做好体检前的各项准备工作, 提高参检人员对体检目的和意义的认识。对个体体检, 在 采集基本信息后, 为其设计科学合理的体检项目。采用体 检与询问调查相结合的方法, 将体检者的基本信息和体检 结果录入深圳“天方达”《杏林妙手》体检软件, 建立健康 档案。诊断标准参照相关部颁标准并在体检软件中设置。 三年间所使用的仪器设备、诊断试剂、检查方法及操作者 均相对固定。

第二步，体检过程中，严格执行各项工作制度和相关 规定。在健康体检的同时, 指导、帮助体检者了解自己的 身体状况, 及时发现部分病患苗头, 建立健康的生活方式。 体检后通过体检软件分析体检者的体格检查、实验室检查 和影像检查结果, 并结合既往史、家族史、生活方式等为 体检者提供详细的健康体检报告。实行重大疾病48 小时内 预警制度, 由专人及时通知体检者或家属, 使体检者能够 在第一时间了解自己的检查结果, 并得到及时的救治。

第三步, 每天下午由各科专家为体检者解读体检报 告、进行咨询答疑, 提出合理的健康建议。团体体检结 束后, 根据需要派出专家上门进行健康咨询、专题讲座 和健康宣教。发现重大疾病者, 则依托医院的医疗优势, 通过绿色通道及时诊治。

第四步, 定期进行电话回访, 提供科学合理个性化的 健康指导, 调动自我监督监测的积极性。督促戒烟限酒、 合理膳食、适量运动, 养成良好的生活习惯; 督促慢性病 患者及时就医并按时按量服药, 保持心态平衡, 将体重、 血压、血糖、血脂等控制在理想范围内。

第五步，从体检软件中提取符合入选条件的体检者 1326 人, 其中男性 875 人, 女性 451 人。统计分析健康管理 前（2015年）和健康管理后（2016年、2017年）体重、血 压、血糖、血尿酸和血脂检查结果的变化情况。

第六步, 所有数据均经SPSS17.0统计软件进行分析处 理, 计数资料以人数 $(n)$ 、百分数 $(\%)$ 表示, 采用 $\chi^{2}$ 检验, 以 $P<0.05$ 为差异有统计学意义。

\section{3. 结果}

\section{1. 体检指标均正常人数}

2015年1月～2017年9月，通过健康管理，各项体检指 标均在正常范围的人数由 192 人上升到 243 人, 体检指标正 常者比例由 $14.48 \%$ 上升到 $18.33 \%$ 。2016年与 2015 年数据相 
比, $\chi^{2}=4.069, P<0.05 ; 2017$ 年与 2015 年数据相比, $\chi^{2}=7.153$, $P<0.01$ 。见表1。

表1 健康管理前后各项体检指标均正常人数 $[n(\%)]$ 。

\begin{tabular}{llll}
\hline 年份 & 2015年 & 2016年 & 2017年 \\
\hline 总人数 & 1326 & 192 & 14.48 \\
各项指标均正常人数 $(n)$ & 1326 & 230 & 17.35 \\
各项指标均正常比例 $(\%)$ & 1326 & 243 & 18.33 \\
\hline
\end{tabular}

\section{2. 体检指标改变}

通过健康管理, 超重、高血压、糖代谢异常、高尿 酸血症和高脂血症的检出率逐年下降，2016年与2015年 比较, 高血压和糖代谢异常的检出率下降明显, 差异有 统计学意义 $(P<0.05) ; 2017$ 年与 2015 年比较, 超重、 高血压、糖代谢异常、高尿酸血症和高脂血症的检出率 均明显下降, 差异有统计学意义 $(P<0.05)$ 。见表2。

表2 健康管理前后各项体检指标改变情况比较 $[n(\%)]$ 。

\begin{tabular}{|c|c|c|c|c|c|c|c|}
\hline & 2015年 & 2016年 & 2017年 & $\chi^{2}{ }_{1}$ 值 & $\chi^{2}{ }_{2}$ 值 & $P_{1}$ 值 & $P_{2}$ 值 \\
\hline 总人数 & 1326 & 1326 & 1326 & & & & \\
\hline 超重 & 211(15.91) & $183(13.80)$ & $175(13.20)$ & 2.337 & 3.929 & $>0.05$ & $<0.05$ \\
\hline 高血压 & $79(5.96)$ & $56(4.22)$ & $32(2.41)$ & 4.129 & 20.770 & $<0.05$ & $<0.01$ \\
\hline 糖代谢异常 & $96(7.24)$ & $68(5.13)$ & $46(3.47)$ & 5.096 & 18.602 & $<0.05$ & $<0.01$ \\
\hline 高尿酸血症 & $137(10.33)$ & $121(9.13)$ & $99(7.47)$ & 1.099 & 6.716 & $>0.05$ & $<0.01$ \\
\hline 高脂血症 & $145(10.94)$ & $135(10.18)$ & $113(8.52)$ & 0.399 & 4.397 & $>0.05$ & $<0.05$ \\
\hline
\end{tabular}

$\chi_{1}^{2}$ 和 $P_{1}: 2016$ 年与 2015 年比较, $\chi_{2}^{2}{ }_{2}$ 和 $P_{2}: 2017$ 年与 2015 年比较

\section{3. 生活方式和治疗依从性改变}

通过健康教育、健康指导和电话回访, 吸烟、酗酒、 运动不足和不合理膳食等不健康行为得到不同程度的改 善, 2016年与 2015 年比较, 差异无统计学意义 $(P>0.05)$,
2017 年与 2015 年比较, 差异有统计学意义 $(P<0.05)$ 。 高血压、糖代谢异常和高脂血症患者服药治疗依从性提 高, 差异有统计学意义 $(P<0.05)$ 。见表3。

表3 健康管理前后不良生活行为改善情况比较 [n（\%)]。

\begin{tabular}{|c|c|c|c|c|c|c|c|}
\hline & 2015年 & 2016年 & 2017年 & $\chi^{2}{ }_{1}$ 值 & $\chi^{2}{ }_{2}$ 值 & $P_{1}$ 值 & $P_{2}$ 值 \\
\hline 总人数 & 1326 & 1326 & 1326 & & & & \\
\hline 吸烟 & $115(8.67)$ & $93(7.01)$ & $86(6.49)$ & 2.525 & 4.527 & $>0.05$ & $<0.05$ \\
\hline 酗酒 & $86(6.49)$ & $75(5.66)$ & $61(4.60)$ & 0.800 & 4.501 & $>0.05$ & $<0.05$ \\
\hline 运动不足 & $430(32.43)$ & $401(30.24)$ & $372(28.05)$ & 1.474 & 6.013 & $>0.05$ & $<0.05$ \\
\hline 不合理膳食 & $372(28.05)$ & $341(25.72)$ & $326(24.59)$ & 1.843 & 4.114 & $>0.05$ & $<0.05$ \\
\hline 治疗依从性 & $101(7.62)$ & $130(9.80)$ & $135(10.18)$ & 3.988 & 5.377 & $<0.05$ & $<0.05$ \\
\hline
\end{tabular}

$\chi_{1}^{2}$ 和 $P_{1}: 2016$ 年与 2015 年比较, $\chi_{2}^{2}$ 和 $P_{2}: 2017$ 年与 2015 年比较

\section{4. 讨论}

\section{1 . 健康管理简单有效}

本研究入选的体检者经过两年的健康管理, 超重、 高血压、糖代谢异常、高尿酸血症、高脂血症的检出率 比管理前明显下降, 差异有统计学意义 $(P<0.05)$, 与杜翠碧[3]的研究结果一致, 可见健康管理干预能有效 降低肥胖、高血压、糖代谢异常、高尿酸血症和高脂血 症的发生率，有效控制代谢综合征在人群中的发展。经 过健康宣教和指导, 体检者不良生活方式有所改善, 不 良嗜好减少, 饮食结构趋于合理, 自觉运动人数增加, 慢性病服药治疗依从性提高, 差异有统计学意义 $(P<$ 0.05）。由此可见，三甲医院在健康体检的基础上实施 健康管理, 简单易行, 效果显著。

\section{2. 健康管理思路实践}

健康管理是防治结合的新学科, 它所涉及的是人的健 康及健康维护和促进。健康管理的思路和实践可追溯到 19 世纪 20 年代末的美国, 其宗旨是调动各方面包括个人及社 会的积极性, 充分利用有限的资源实现健康效果最大化。
健康管理在发达国家经历多年的发展, 已成为其医疗服务 体系中不可或缺的一部分。人们在健康或者亚健康状态时 就应该进行干预管理, 延迟疾病的发生或发展, 使机体保 持尽可能长的健康状态 [1]。目前, 美国约有 $70 \%$ 的人口都 在健康管理的服务范围内。当今社会工作紧张压力大、生 活节奏快、市场竞争激烈、人际关系复杂，生活方式性疾 病的防治成为健康管理的基本内容。针对体检的结果, 在 健康管理中, 积极实施干预措施, 对高危人群进行非药物 治疗的个体化指导; 对疾病人群改变单纯依靠药物治疗的 传统做法, 采用健康促进诊疗的管理模式, 能充分调动主 观能动性, 提高自我管理能力, 保证长期治疗方案的严格 执行，降低并发症的发生，提高患者的生活质量。

\section{3. 健康管理实施步骤}

健康管理实施的具体步骤包括: 健康状况的信息采 集、健康状况评价和预测、健康促进行为干预及咨询指 导四个部分 [4]。健康管理是一种前瞻性的服务模式, 它 的出现是时代发展的需要, 它通过创造一个推动自我健 康改善的有效环境, 帮助个人建立健康的生活方式, 并 采取行动来降低疾病危险性以达到改善健康的最终目 的, 有效地为体检中心提供了一个与客户保持长期互动 
的平台和通道, 延伸了健康体检中心的服务。健康体检 即健康状况的信息采集是健康管理流程中的第一步骤 [5], 设计科学合理的体检套餐, 是获取科学、准确、有 效健康信息的基础。健康档案要做到数据格式标准化、 规范化、科学化、电子化、网络化, 才能够真正做到可 及可用、便捷高效, 利于分析、发掘和利用 $[6]$ 。健康体 检对疾病的早发现、早预防属于发现问题阶段, 而问题 的解决则需要借助于健康管理工作的开展 [7]。

\section{4. 健康管理前景广阔}

预防医学和健康保健将成为 21 世纪医学的主导。三甲 医院健康体检中心完成由单纯健康体检向健康管理的转 变, 已经成为健康体检行业和体检机构的共识。三甲医院 具有人才、技术、经验、财力等诸多优势, 可提供科学规 范的健康体检服务、全面认真地收集健康信息、建立和维 护好健康档案、科学细致地进行分析评估、切实有效地实 施教育干预、长期连续周而复始地不断服务, 实现健康体 检向健康管理的升级 [6]。三甲医院的品牌影响力有助于获 得社会和客户的认可与信赖, 拥有稳定、庞大的客户群及 潜在客户群, 医院雄厚的实力为开展健康管理服务提供了 有力的保障[8]。三甲医院拥有功能齐全的学科建制, 高层 次的医疗专业队伍和科学先进的医疗设备, 对健康状况的 评估和疾病风险的干预具有权威性。后续治疗便捷, 对健 康体检后所发现的问题可通过绿色通道进行专科就医。有 良好的教育资源和组织开展教育活动的优势, 医疗信息更 及时, 内容更丰富, 可进行针对性教育, 也可进行科普性 教育[9]。健康管理在三甲医院健康体检中具有广泛的应用 前景, 尤其在随访、复诊、用药指导、康复等健康管理时 更具专业性, 发展潜力更大。

\section{5. 健康管理机遇挑战}

健康管理目前缺乏有力的政策支持和经济导向, 实施 驱动力和创新不足, 专业人员和相关技术贵乏, 合理有效 的健康管理模式仍未建立, 全人、全程、全方位的健康管 理服务远远无法达到 [6], 健康管理理论只停留在概念层面 [10], 人性化服务和全程健康指导欠缺 $[1]$ 。健康管理属于 创新医学服务体系, 许多工作需要开拓进取, 三甲医院在 信息系统方面有丰富的创新能力, 运用信息网络技术, 能 更好地提升健康管理的绩效。移动终端的迅速普及, 为健 康管理的发展提供了无限的可能和空间。移动客户端作为 个人健康自我维护和沟通的平台, 为客户提供了预约、咨 询、查询、随访、投诉和监管等功能模块, 使健康管理工 作更科学、规范、深入和有效。健康管理如何利用相关行
业的发展机遇, 为自身学科发展提供强劲的动力, 既是挑 战也是机遇 [6]。只有加强学科建设才能提高核心竞争力, 才能促使健康管理服务更加科学、更加系统、更加长远地 发展, 才能引领健康管理行业规范、健康、有序的发展 [11]。 科学的发展战略、扁平的组织结构、健全的组织制度、良 好的组织文化和合理的人力资源结构能够促进三甲医院 健康管理中心更快更好地发展[12]。

\section{参考文献}

[1] 王野虹, 仵淑娟, 徐红. 发挥综合医院特长深化健康管理内 涵[J]. 中国疗养医学, 2014,23(10):954-956

[2] 周晓丹, 施洪, 李贺, 黄林. 基于健康体检某部队干部的健 康管理与效果评价 [J]. 中华保健医学杂志, 2015,17(4):323-324

[3] 杜翠碧. 健康管理干预对健康体检人群代谢综合症发生情 况的影响 [J]. 中国卫生标准管理，2015(22):18-20

[4] 陈君石, 黄建始. 健康管理师[M]. 北京: 中国协和医科大学 出版社, 2007,12

[5] 王晶晶. 从健康体检到健康管理的探讨 [J]. 甘肃科技, 2016,32(21):70-71

[6] 安辉, 李幼林, 曹晓华. 综合医院实施健康管理的实践探讨 [J]. 医院管理论坛，2015,32(11):57-59

[7] 张娟, 刘敏. 健康体检发展与健康管理的模式分析 [J]. 中国 医药指南, 2015,13(33):297-298

[8] 文浩太, 王巧莲, 南培宏. 大型综合医院开展健康管理服务 的SWOT分析[J]. 卫生软科学, 2014,28(9):587-589

[9] 汪荷, 黄津芳, 韩悦容. 综合医院健康管理的学科优势与作 用 [J]. 沈阳部队医药, 2012,4(25):278-280

[10] 王仲. 对新医改下病人就医需求的认识与思考 $[\mathrm{J}]$. 中国医院 管理， 2014,34(4):4-5

[11] 王怡丹, 吴云车, 李景波. 基于SWOT法三级综合医院健康 管理中心发展策略研究 [J]. 中国医院管理, 2015,35(12):97-99

[12] 王怡丹, 赵小兰, 李景波, 吴云车. 基于麦肯锡 $7 \mathrm{~S}$ 模型的综 合医院健康管理中心现状调查 [J]. 中国医院管理, 2016,36(4):75-77 